\title{
TOPOLOGICAL TYPES AND MULTIPLICITIES OF ISOLATED QUASI-HOMOGENEOUS SURFACE SINGULARITIES
}

\author{
STEPHEN S.-T. YAU
}

\begin{abstract}
Two germs of 2-dimensional isolated quasi-homogeneous hypersurface singularities have the same topological type if and only if they have the same characteristic polynomial and the same fundamental group for their links. In particular, multiplicity is an invariant of topological type, an affirmative answer to Zariski's question in this case.
\end{abstract}

Let $(V, 0)$ and $(W, 0)$ be germs of isolated hypersurface singularities in $\mathbf{C}^{n+1}$. We say that $(V, 0)$ and $(W, 0)$ have the same topological type if there is a germ of a homeomorphism from $\left(\mathbf{C}^{n+1}, V, 0\right)$ to $\left(\mathbf{C}^{n+1}, W, 0\right)$. In his retiring Presidential address to the American Mathematical Society in 1971, Zariski asked whether $(V, 0)$ and $(W, 0)$ have the same multiplicity if they have the same topological type. He expected that topologists would be able to answer his question in relatively short order. However the question appears to be much harder than what Zariski thought. Even special cases of Zariski's problem have proved to be extremely difficult. Only recently Greuel [4] and O'Shea [14] proved independently that topological type constant families of isolated quasi-homogeneous singularities are equimultiple. For quasihomogeneous surface singularities, Laufer [5] explained the constant multiplicity for a topological type constant family of singularities from a different viewpoint. However it is not known whether two quasi-homogeneous singularities having the same topological type can be put into a topological type constant family. Let $(V, 0)$ be a dimension two isolated hypersurface singularity. Lê and Teissier [8] observed that A'Campo's work [1] can often be used to give positive results towards Zariski's question. Let $C(V, 0)$ be the reduced tangent cone. Let $\mathbf{P} C(V, 0)$ denote the hypersurface in $\mathbf{C P}^{2}$ over which $C(V, 0)$ is a cone. Then, the work of A'Campo shows that the multiplicity of $(V, 0)$ is determined by the topological type of $(V, 0)$ in case the topological Euler number $\chi(\mathbf{P} C(V, 0))$ is nonzero. The same arguments also show that, for isolated hypersurface two-dimensional singularities, the embedded topology and the multiplicity determine $\chi(\mathbf{P} C(V, 0)))$. However, so far, by using A'Campo's result, one can only prove that a surface in $\mathbf{C}^{3}$ having at 0 a singularity of multiplicity 2 cannot have the same topological type at 0 as another surface of multiplicity different from 2 .

Received by the editors March 23, 1988.

1980 Mathematics Subject Classification (1985 Revision). Primary 32B99; Secondary 32C40. This research is partially supported by N. S. F. Grant No. DMS 8411477. 
For plane curve singularities, the Zariski question was known to be true. The reason that the Zariski question could be answered was that the topological types of plane curve singularities were well understood (see $[\mathbf{2}, \mathbf{3}, \mathbf{9}, \mathbf{1 6}$, 19, 22]).

Let $(V, 0)$ be an isolated hypersurface singularity in $\mathbf{C}^{n+1}$ defined by the holomorphic function $f:\left(\mathbf{C}^{n+1}, 0\right) \rightarrow(\mathbf{C}, 0)$. Then the link of $(V, 0)$ is $K_{V}=$ $S_{\varepsilon}^{2 n+1} \cap V$ where $S_{\varepsilon}^{2 n+1}$ is the $(2 n+1)$-sphere of radius $\varepsilon$ centered at the origin and $\varepsilon>0$ is sufficiently small. It is known that $\pi_{1}\left(K_{V}\right)$ depends only on the topological type of $(V, 0)[6,10]$.

Let $0<\delta \ll \varepsilon \ll 1$. We define

$$
\begin{gathered}
T=\{t \in \mathbf{C}:|t|<\delta\}, \quad B=\left\{z \in \mathbf{C}^{n+1}:\left|z_{0}\right|^{2}+\cdots+\left|z_{n}\right|^{2}<\varepsilon\right\}, \\
X=B \cap f^{-1}(T), \quad X(t)=B \cap f^{-1}(t) .
\end{gathered}
$$

Milnor proved that $f: X \backslash X(0) \rightarrow T \backslash\{0\}$ is a locally trivial smooth fiber bundle and the fiber has the homotopy type of a bouquet of $n$-spheres. The generator of $\pi_{1}(T \backslash\{0\})$ (represented by a counter-clockwise oriented circle around the origin) induces the monodromy automorphism $h: H^{*}(X(t), \mathbf{C}) \rightarrow$ $H^{*}(X(t), \mathbf{C})$. Let $\Delta_{V}(z)$ denote the characteristic polynomial $\Delta_{V}(z)=$ $\operatorname{det}\left(z I-h^{*}\right)$ of the linear transformation $h^{*}: H^{n}(X(t), \mathbf{C}) \rightarrow H^{n}(X(t), \mathbf{C})$; $\Delta_{V}(z)$ is an invariant of topological type of $(V, 0)[6]$. Recall that a hypersurface singularity $(V, 0)=\left\{\left(z_{0}, \ldots, z_{n}\right): f\left(z_{0}, \ldots, z_{n}\right)=0\right\} \subseteq \mathbf{C}^{n+1}$ is quasihomogeneous if $f$ is in the Jacobian ideal of $f$, i.e., $f \in\left(\partial f / \partial z_{0}, \ldots, \partial f / \partial f_{n}\right)$. The main purpose of this note is to announce the following results.

THEOREM A. Let $(V, 0)$ and $(W, 0)$ be two isolated quasi-homogeneous surface singularities in $\mathbf{C}^{3}$. Then $\left(\mathbf{C}^{3}, V, 0\right)$ is homeomorphic to $\left(\mathbf{C}^{3}, W, 0\right)$ if and only if $\pi_{1}\left(K_{V}\right) \cong \pi_{1}\left(K_{W}\right)$ and $\Delta_{V}(z)=\Delta_{W}(z)$.

A polynomial $h\left(z_{0}, \ldots, z_{n}\right)$ is weighted homogeneous of type $\left(w_{0}, \ldots, w_{n}\right)$, where $\left(w_{0}, \ldots, w_{n}\right)$ are fixed positive rational numbers, if it can be expressed as a linear combination of monomials $z_{0}^{i_{0}} z_{0}^{i_{1}} \cdots z_{n}^{i_{n}}$ for which $i_{0} / w_{0}+\cdots+$ $i_{n} / w_{n}=1$. $\left(w_{0}, \ldots, w_{n}\right)$ is called the weights of $h$. As a corollary to the proof of Theorem A, we have the following corollary.

THEOREM B. Let $(V, 0)$ be an isolated quasi-homogeneous surface singularity defined by a weighted homogeneous polynomial in $\mathbf{C}^{3}$ with weights $\left(w_{0}, w_{1}, w_{2}\right)$. Then the topological type of $(V, 0)$ determines and is determined by its weights $\left(w_{0}, w_{1}, w_{2}\right)$.

The following theorem solves the Zariski question completely in the case of quasi-homogeneous surface singularities in $\mathbf{C}^{3}$.

THEOREM C. Let $(V, 0)$ and $(W, 0)$ be two isolated quasi-homogeneous surface singularities in $\mathbf{C}^{3}$. If $\left(\mathbf{C}^{3}, V, 0\right)$ is homeomorphic to $\left(\mathbf{C}^{3}, W, 0\right)$ as a germ, then $V$ and $W$ have the same multiplicity at the origin.

Sketch of The Proof of Theorem A. Suppose that a real manifold $B$ of dimension $m$ and a family $\left\{\left(M_{t}, N_{t}\right): t \in B, N_{t}\right.$ is a closed submanifold of a compact differentiable manifold $M_{t}$ \} are given. We say that $\left(M_{t}, N_{t}\right)$ 
depends $C^{\infty}$ on $t$ and that $\left\{\left(M_{t}, N_{t}\right): t \in B\right\}$ is a $C^{\infty}$ family of compact manifolds with submanifolds if there is a $C^{\infty} \mathscr{M}$, closed submanifold $\mathscr{N}$, and a $C^{\infty}$ map $\omega$ from $\mathscr{M}$ onto $B$ such that $\bar{\omega}=\omega / \mathscr{N}$ is also a $C^{\infty}$ map from $\mathscr{N}$ onto $B$ satisfying the following conditions:

(i) $M_{t}=\omega^{-1}(t) \supseteq N_{t}=\bar{\omega}^{-1}(t)$.

(ii) The rank of the Jacobian of $\omega$ (respectively $\bar{\omega}$ ) is equal to $m$ at every point of $\mathscr{M}$ (respectively $\mathscr{N}$ ).

We first prove the following theorem which is essential in proving the above theorems. The theorem is classically known when $\mathscr{N}$ is an empty set.

THEOREM 1. Let $((\mathscr{M}, \mathscr{N}),(\omega, \bar{\omega}), B)$ be a $C^{\infty}$ family of compact manifolds with submanifolds, and $t_{0}$ any point of $B$. Then

$$
\left(M_{t}, N_{t}\right)=\left(\omega^{-1}(t), \bar{\omega}^{-1}(t)\right)
$$

is diffeomorphic to $\left.\left(M_{t_{0}}, N_{t_{0}}\right)=\left(\omega^{-1}\left(t_{0}\right), \bar{\omega}^{-1} t_{0}\right)\right)$ for any $t \in B$ if $B$ is connected.

It follows from the above theorem that we have:

THEOREM 2. Suppose $f\left(z_{0}, z_{1}, z_{2}\right)$ and $g\left(z_{0}, z_{1}, z_{2}\right)$ are weighted homogeneous polynomials with the same weights $\left(w_{0}, w_{1}, w_{2}\right)$. If the variety $V$ of $f$ and the variety $W$ of $g$ have isolated singularity at the origin, then $\left(\mathbf{C}^{3}, V, 0\right)$ is homeomorphic equivalent to $\left(\mathbf{C}^{3}, W, 0\right)$.

In order to see Theorem 2 , we let $\Delta$ be the intersection of the plane $x / w_{0}+y / w_{1}+z / w_{2}=1$ with the first quadrant of $\mathbf{R}^{3}$. Let $N$ be the number of integral points $\left(\alpha_{0}, \alpha_{1}, \alpha_{2}\right)$ which are in $\Delta$. Let $F=\sum a_{\left(\alpha_{0}, \alpha_{1}, \alpha_{2}\right)} z_{0}^{\alpha_{0}} z_{1}^{\alpha_{1}} z_{2}^{\alpha_{2}}$, where the summation is over all the integral points in $\Delta$. Then $F$ is a complete family of weighted homogeneous polynomials with weights $\left(w_{0}, w_{1}, w_{2}\right)$. Let $\mathscr{V}=\left\{\left(z_{0}, z_{1}, z_{2}, \ldots, a_{\left(\alpha_{0}, \alpha_{1}, \alpha_{2}\right)}, \ldots\right): F=0\right\}$ be a variety of $\mathbf{C}^{N+3}$ defined by $F=0$. Consider the natural projection $\pi$ from $\mathbf{C}^{N+3}$ to $\mathbf{C}^{N}$ given by sending $\left(z_{0}, z_{1}, z_{2}, \ldots, a_{\left(\alpha_{0}, \alpha_{1}, \alpha_{2}\right)}, \ldots\right)$ to $\left(\ldots, a_{\left(\alpha_{0}, \alpha_{1}, \alpha_{2}\right)}, \ldots\right)$ and $\bar{\pi}$ be the restriction of $\pi$ to $\mathscr{V}$. Let $B$ be the set of all $\left(\ldots, a_{\left(\alpha_{0}, \alpha_{1}, \alpha_{2}\right)}, \ldots\right)$ in $\mathbf{C}^{N}$ such that $\pi^{-1}\left(\ldots, a_{\left(\alpha_{0}, \alpha_{1}, \alpha_{2}\right)}, \ldots\right)=\left\{\left(z_{0}, z_{1}, z_{2}\right): \sum a_{\left(\alpha_{0}, \alpha_{1}, \alpha_{2}\right)} z_{0}^{\alpha_{0}} z_{1}^{\alpha_{1}} z_{2}^{\alpha_{2}}=0\right\}$ has isolated singularity at the origin. Let $\mathscr{M}=\left\{\left(z_{0}, z_{1}, z_{2}, \ldots, a_{\left(\alpha_{0}, \alpha_{1}, \alpha_{2}\right)}, \ldots\right)\right.$ : $\left.\left|z_{0}\right|^{2}+\left|z_{1}\right|^{2}+\left|z_{2}\right|^{2}=1\right\}$ and $\mathscr{N}=\mathscr{V} \cap \mathscr{M}$. Denote $\omega$ and $\bar{\omega}$ be the restriction of $\pi$ and $\bar{\pi}$. Then one checks that the conditions in Theorem 1 are satisfied.

It follows from Theorem 2 and an argument due to Orlik and Wagreich [13] (see also Arnold [0]) that any isolated singularity $(V, 0)$ in $\mathbf{C}^{3}$ defined by a weighted homogeneous polynomial has the same topological type as one of the following seven classes of singularities with the same weights as $(V, 0)$.

Class I: $V\left(a_{0}, a_{1}, a_{2} ; I\right)=\left\{z_{0}^{a_{0}}+z_{1}^{a_{1}}+z_{2}^{a_{2}}=0\right\}$.

Class II: $V\left(a_{0}, a_{1}, a_{2} ; I I\right)=\left\{z_{0}^{a_{0}}+z_{1}^{a_{1}}+z_{1} z_{2}^{a_{2}}=0\right\}, a_{1}>1$.

Class III: $V\left(a_{0}, a_{1}, a_{2} ; I I I\right)=\left\{z_{0}^{a_{0}}+z_{1}^{a_{1}} z_{2}+z_{1} z_{2}^{a_{2}}=0\right\}, a_{1}>1, a_{2}>1$.

Class IV: $V\left(a_{0}, a_{1}, a_{2} ; I V\right)=\left\{z_{0}^{a_{0}}+z_{0} z_{1}^{a_{1}}+z_{1} z_{2}^{a_{2}}=0\right\}, a_{0}>1$.

Class V: $V\left(a_{0}, a_{1}, a_{2} ; V\right)=\left\{z_{0}^{a_{0}} z_{1}+z_{1}^{a_{1}} z_{2}+z_{0} z_{2}^{a_{2}}=0\right\}$.

Class VI: $V\left(a_{0}, a_{1}, a_{2} ; V I\right)=\left\{z_{0}^{a_{0}}+z_{0} z_{1}^{a_{1}}+z_{0} z_{2}^{a_{2}}+z_{1}^{b_{1}} z_{2}^{b_{2}}=0\right\}$, where $\left(a_{0}-1\right)\left(a_{1} b_{2}+a_{2} b_{1}\right)=a_{0} a_{1} a_{2}$. 
Class VII: $V\left(a_{0}, a_{1}, a_{2} ; V I I I\right)=\left\{z_{0}^{a_{0}} z_{1}+z_{0} z_{1}^{a-1}+z_{0} z_{2}^{a_{2}}+z_{1}^{b_{1}} z_{2}^{b_{2}}=0\right\}$, where $\left(a_{0}-1\right)\left(a_{1} b_{2}+a_{2} b_{1}\right)=a_{2}\left(a_{0} a_{1}-1\right)$.

It is well known that any isolated two-dimensional hypersurface quasihomogeneous singularities $(V, 0)$ is actually weighted homogeneous after a biholomorphic change of coordinate. Hence in order to prove Theorem A, we may assume that both $(V, 0)$ and $(W, 0)$ are one of the seven classes above. Now we need two important results due to Neumann [12] and Orlik-Wagreich [13] about the abstract topology of these singularities. Neumann's results say that the minimal resolutions of these singularities are determined by the fundamental groups. Orlik-Wagreich computed the minimal resolutions of these singularities explicitly in terms of their weights. On the other hand Milnor-Orlik [11] and Varchenko [17] computed the monodromy of these singularities explicitly. Yoshinaga [18] observed that the Milnor-Orlik result has strong consequences on $u_{i}$ once we know the characteristic polynomial of the singularity, where $u_{i} / v_{i}$ is a reduced fraction of the weight $w_{i}$. Varchenko's result allows us to write down the characteristic polynomials of the above seven class singularities in terms of $a_{0}, a_{1}$, and $a_{2}$ explicitly. By using the explicit data of the minimal resolutions and characteristic polynomials, we prove that if $(V, 0)$ and $(W, 0)$ are one of the seven classes and they have the same characteristic polynomials and fundamental groups, then they have the same weights (see Theorem 3 below for a more precise and stronger statement). It follows from Theorem 2 that $(V, 0)$ and $(W, 0)$ have the same topological type. This completes the proof of Theorem A.

PROOF OF THEOREM B. By Theorem A, the topological type of $(V, 0)$ determines the characteristic polynomial and the fundamental group of the singularity. However in the course of proving Theorem A, we have shown that the characteristic polynomial and the fundamental group of the singularity determine its weights. Conversely, the weights determine the topological type of the singularity by Theorem 2 .

In fact, Theorem 3 is interesting in its own right.

THEOREM 3. Let $f$ and $g$ be weighted homogeneous polynomials with isolated critical points at the origin. Suppose that $\Delta_{f}(z)=\Delta_{g}(z)$ and $\pi_{1}\left(K_{f}\right)=$ $\pi_{1}\left(K_{g}\right)$.

(I) If both $f$ and $g$ are of class I, then $f$ and $g$ have the same weights and

$$
\left\{\begin{array}{l}
f=z_{0}^{a_{i_{0}}}+z_{1}^{a_{i_{1}}} 1+z_{2}^{a_{i_{2}}}, \\
g=z_{0}^{a_{0}}+z_{1}^{a_{1}}+z_{2}^{a_{2}},
\end{array}\right.
$$

where $\left\{i_{0}, i_{1}, i_{2}\right\}=\{0,1,2\}$.

(II) If $f$ and $g$ are both of class II, then $f$ and $g$ have the same weights and either

$$
f=z_{0}^{a_{0}}+z_{1}^{a_{1}}+z_{1} z_{2}^{a_{2}}=g
$$

or

$$
\left\{\begin{array}{l}
f=z_{0}^{a_{1}}+z_{1}^{a_{0}}+z_{1} z_{2}^{\frac{a_{2} a_{1}\left(a_{0}-1\right)}{a_{0}\left(a_{1}-1\right)}} \\
g=z_{0}^{a_{0}}+z_{1}^{a_{1}}+z_{1} z_{2}^{a_{2}}
\end{array}\right.
$$


with $a_{0}\left(a_{1}-1\right)$ divides $a_{1} a_{2}\left(a_{0}-1\right)$, or

$$
\left\{\begin{array}{l}
f=z_{0}^{m(k+1)}+z_{1}^{k+1}+z_{1} z_{2}^{n k} \\
g=z_{0}^{n(k+1)}+z_{1}^{k+1}+z_{1} z_{2}^{m k}
\end{array}\right.
$$

(III) If $f$ and $g$ are both of class III, then $f$ and $g$ have the same weights and either

$$
f=z_{0}^{a_{0}}+z_{1}^{a_{1}} z_{2}+z_{1} z_{2}^{a_{2}}=g
$$

or

$$
\left\{\begin{array}{l}
f=z_{0}^{a_{0}}+z_{1}^{a_{2}} z_{2}+z_{1} z_{2}^{a_{1}}, \\
g=z_{0}^{a_{0}}+z_{1}^{a_{1}} z_{2}+z_{1} z_{2}^{a_{2}} .
\end{array}\right.
$$

(IV) If $f$ and $g$ are both of class IV, then

$$
f=z_{0}^{a_{0}}+z_{0} z_{1}^{a_{1}}+z_{1} z_{2}^{a_{2}}=g .
$$

(V) If $f$ and $g$ are both of class $\mathrm{V}$, then $f$ and $g$ have the same weights and either

$$
\left\{\begin{array}{l}
f=z^{a_{i_{0}}} z_{1}+z_{1}^{a_{i_{1}}} z_{2}+z_{0} z_{2}^{a_{i_{2}}}, \\
g=z_{0}^{a_{0}} z_{1}+z_{1}^{a_{1}} z_{2}+z_{0} z_{2}^{a_{2}},
\end{array}\right.
$$

where $\left\{i_{0}, i_{1}, i_{2}\right\}$ is an even permutation of $\{0,1,2\}$ or

$$
\left\{\begin{array}{l}
f=z_{1} z_{0}^{\frac{a_{1}\left(a_{0} a_{2}-a_{0}+1\right)}{a_{1} a_{2}-a_{2}+1}}+z_{2} z_{1}^{\frac{a_{0}\left(a_{1} a_{2}-a_{2}+1\right)}{\left(a_{0} a_{1}-a_{1}+1\right)}}+z_{0} z_{2}^{\frac{a_{2}\left(a_{0} a_{1}-a_{1}+1\right)}{\left(a_{0} a_{2}-a_{0}+1\right)}} \\
g=z_{0}^{a_{0}} z_{1}+z_{1}^{a_{1}} z_{2}+z_{0} z_{2}^{a_{2}}
\end{array}\right.
$$

with all the exponents being integers, or

$$
\left\{\begin{array}{l}
f=z_{1} z_{0}^{\frac{a_{0}\left(a_{1} a_{2}-a_{2}+1\right)}{a_{0} a_{1}-a_{1}+1}}+z_{2} z_{1}^{\frac{a_{2}\left(a_{0} a_{1}-a_{1}+1\right)}{a_{0} a_{2}-a_{0}+1}}+z_{0} z_{2}^{\frac{a_{1}\left(a_{0} a_{2}-a_{0}+1\right)}{a_{1} a_{2}-a_{2}+1}} \\
g=z_{0}^{a_{0}} z_{1}+z_{1}^{a_{1}} z_{2}+z_{0} z_{2}^{a_{2}}
\end{array}\right.
$$

with all the exponents being integers, or

$$
\left\{\begin{array}{l}
f=z_{1} z_{0}^{\frac{a_{2}\left(a_{0} a_{1}-a_{1}+1\right)}{a_{0} a_{2}-a_{0}+1}}+z_{2} z_{1}^{\frac{a_{1}\left(a_{0} a_{2}-a_{0}+1\right)}{a_{1} a_{2}-a_{2}+1}}+z_{0} z_{2}^{\frac{a_{0}\left(a_{1} a_{2}-a_{2}+1\right)}{a_{0} a_{1}-a_{1}+1}}, \\
g=z_{0}^{a_{0}} z_{1}+z_{1}^{a_{1}} z_{2}+z_{0} z_{2}^{a_{2}}
\end{array}\right.
$$

with all the exponents being integers.

(VI) If $f$ and $g$ are both of type VI, then $f$ and $g$ have the same weights and either

$$
\left\{\begin{array}{l}
f=z_{0}^{a_{0}}+z_{0} z_{1}^{a_{1}}+z_{0} z_{2}^{a_{2}}+z_{1}^{\bar{b}_{1}} z_{2}^{\bar{b}_{2}}, \\
g=z_{0}^{a_{0}}+z_{0} z_{1}^{a_{1}}+z_{0} z_{2}^{a_{2}}+z_{1}^{b_{1}} z_{2}^{b_{2}}
\end{array}\right.
$$

or

$$
\left\{\begin{array}{l}
f=z_{0}^{a_{0}}+z_{0} z_{1}^{a_{2}}+z_{0} z_{2}^{a_{1}}+z_{1}^{\bar{b}_{1}} z_{2}^{\bar{b}_{2}}, \\
g=z_{0}^{a_{0}}+z_{0} z_{1}^{a_{1}}+z_{0} z_{2}^{a_{2}}+z_{1}^{b_{1}} z_{2}^{b_{2}} .
\end{array}\right.
$$

(VII) If $f$ and $g$ are both of type VII, then $f$ and $g$ have the same weights and either

$$
\left\{\begin{array}{l}
f=z_{0}^{a_{0}} z_{1}+z_{0} z_{1}^{a_{1}}+z_{0} z_{2}^{a_{2}}+z_{1}^{\bar{b}_{1}} z_{2}^{\bar{b}_{2}} \\
g=z_{0}^{a_{0}} z_{1}+z_{0} z_{1}^{a_{1}}+z_{0} z_{2}^{a_{2}}+z_{1}^{b_{1}} z_{2}^{b_{2}}
\end{array}\right.
$$


or

$$
\left\{\begin{array}{l}
f=z_{0}^{a_{1}} z_{1}+z_{0} z_{1}^{a_{0}}+z_{0} z_{2}^{\frac{a_{0} a_{2}\left(a_{1}-1\right)}{a_{1}\left(a_{0}-1\right)}}+z_{1}^{\bar{b}_{2}} z_{2}^{\bar{b}_{2}}, \\
g=z_{0}^{a_{0}} z_{1}+z_{0} z_{1}^{a_{1}}+z_{0} z_{2}^{a_{2}}+z_{1}^{b_{1}} z_{2}^{b_{2}}
\end{array}\right.
$$

with $a_{1}\left(a_{0}-1\right)$ divides $a_{0} a_{2}\left(a_{1}-1\right)$, or

$$
\left\{\begin{array}{l}
f=z_{1} z_{0}^{\frac{a_{2}\left(a_{0} a_{1}-1\right)-a_{1}\left(a_{0}-1\right)}{a_{2}\left(a_{0}-1\right)}}+z_{0} z_{1}^{\frac{a_{0} a_{2}\left(a_{1}-1\right)}{a_{1}\left(a_{0}-1\right)}}+z_{0} z_{2}^{a_{0}}+z_{1}^{\bar{b}_{1}} z_{2}^{\bar{b}_{2}}, \\
g=z_{0}^{a_{0}} z_{1}+z_{0} z_{1}^{a_{1}}+z_{0} z_{2}^{a_{2}}+z_{1}^{b_{1}} z_{2}^{b_{2}}
\end{array}\right.
$$

with all the exponents being integers and $a_{2}$ divides $a_{1}\left(a_{0}-1\right) /\left(a_{0}-1, a_{1}-1\right)$ where $(a, b)$ denotes the greatest common divisor of $a$ and $b$, or

$$
\left\{\begin{array}{l}
f=z_{0}^{a_{2}} z_{1}+z_{0} z_{1}^{\frac{a_{2}\left(a_{0} a_{1}-1\right)-a_{1}\left(a_{0}-1\right)}{a_{2}\left(a_{1}-1\right)}}+z_{0} z_{2}^{\frac{a_{2}\left(a_{0} a_{1}-1\right)-a_{1}\left(a_{0}-1\right)}{a_{2}\left(a_{0}-1\right)}}+z_{1}^{\bar{b}_{1}} z_{2}^{\bar{b}_{2}}, \\
g=z_{0}^{a_{0}} z_{1}+z_{0} z_{1}^{a_{1}}+z_{0} z_{2}^{a_{2}}+z_{1}^{b_{1}} z_{2}^{b_{2}}
\end{array}\right.
$$

with all the exponents being integers and $a_{2}$ divides $a_{1}\left(a_{0}-1\right) /\left(a_{0}-1, a_{1}-1\right)$, or

$$
\left\{\begin{array}{l}
f=z_{1} z_{0}^{\frac{a_{2}\left(a_{0} a_{1}-1\right)-a_{1}\left(a_{0}-1\right)}{a_{2}\left(a_{1}-1\right)}}+z_{0} z_{1}^{a_{2}}+z_{0} z_{2}^{a_{1}}+z_{1}^{\bar{b}_{1}} z_{2}^{\bar{b}_{2}}, \\
g=z_{0}^{a_{0}} z_{1}+z_{0} z_{1}^{a_{1}}+z_{0} z_{2}^{a_{2}}+z_{1}^{b_{1}} z_{2}^{b_{2}}
\end{array}\right.
$$

with all exponents being integers and $a_{2}$ divides $a_{1}\left(a_{0}-1\right) /\left(a_{0}-1, a_{1}-1\right)$, or (6)

$\left\{\begin{array}{l}f=z_{1} z_{2}^{\frac{a_{0} a_{2}\left(a_{1}-1\right)}{a_{1}\left(a_{0}-1\right)}}+z_{0} z_{1}^{\frac{a_{2}\left(a_{0} a_{1}-1\right)-a_{1}\left(a_{0}-1\right)}{a_{2}\left(a_{0}-1\right)}}+z_{0} z_{2}^{\frac{a_{2}\left(a_{0} a_{1}-1\right)-a_{1}\left(a_{0}-1\right)}{a_{2}\left(a_{1}-1\right)}}+z_{1}^{\bar{b}_{1}} z_{2}^{\bar{b}_{2}} \\ g=z_{0}^{a_{0}} z_{1}+z_{0} z_{1}^{a_{1}}+z_{0} z_{2}^{a_{2}}+z_{1}^{b_{1}} z_{2}^{b_{2}}\end{array}\right.$

with all the exponents being integers and $a_{2}$ divides $a_{1}\left(a_{0}-1\right) /\left(a_{0}-1, a_{1}-1\right)$.

There are analogous theorems in case $f$ and $g$ are in different classes.

PROOF OF THEOREM C. We first observe the following lemma.

LEMMA 4. Let $f\left(z_{0}, z_{1}, z_{2}\right)$ be a weighted homogeneous polynomial with weights $\left(w_{0}, w_{1}, w_{2}\right)$. Suppose that $f$ has an isolated singularity at the origin. Denote the multiplicity of $f$ at the origin by $m_{f}$. Then $m_{f} \geq \min \left\{w_{0}, w_{1}, w_{2}\right\}$.

We next prove the following proposition:

Proposition 5. Suppose that $f$ is one of the seven classes. Let $m=$ $\min \left\{n \in \mathbf{Z}: n \geq \min \left\{w_{0}, w_{1}, w_{2}\right\}\right\}$. Then the multiplicity of $m_{f}$ of $f$ is $m$.

Because of Theorem B, we only need to prove Theorem 6 in order to prove Theorem C.

THEOREM 6. Let $f\left(z_{0}, z_{1}, z_{2}\right)$ be a weighted homogeneous polynomial with weights $\left(w_{0}, w_{1}, w_{2}\right)$. Suppose that $f$ has an isolated singularity at the origin. Then $m_{f}$, the multiplicity of $f$ at the origin, equals

$$
\min \left\{m \in \mathbf{Z}: m \geq \min \left\{w_{0}, w_{1}, w_{2}\right\}\right\} .
$$

ProOF. By Orlik-Wagreich $[\mathbf{1 3}], f$ can be written as $g+h$, where $g\left(z_{0}, z_{1}, z_{2}\right)$ is one of the seven classes having the same weights $\left(w_{0}, w_{1}, w_{2}\right)$ as $f$, and $g$ and $h$ have no monomial term in common. It is clear that $m_{f} \leq m_{g}$. By 
Proposition 5, we have $m_{g}=\min \left\{n \in \mathbf{Z}: n \geq \min \left\{w_{0}, w_{1}, w_{2}\right\}\right\}$. Hence we deduce that $m_{f} \leq \min \left\{n \in \mathbf{Z}: n \geq \min \left\{w_{0}, w_{1}, w_{2}\right\}\right\}$.

Conversely, by Lemma 4 , we see that $m_{f} \geq \min \left\{w_{0}, w_{1}, w_{2}\right\}$. Thus $m_{f} \geq$ $\min \left\{n \in \mathbf{Z}: n \geq \min \left\{w_{0}, w_{1}, w_{2}\right\}\right\}$. Q.E.D.

ADDED IN PROOF. The details of the proofs had been written up jointly with Yijing $\mathrm{Xu}$ and is available in preprint form. We are currently making progress on those singularities whose Newton diagrams consist of two compact facets.

\section{REFERENCES}

0. V. I. Arnold, Normal forms of functions in neighborhood of degenerate critical points, Russian Math. Surveys 29 (1975), 10-150.

1. N. A'Campo, La fonction zeta d'une monodromie, Comment. Math. Helv. 50 (1975), 233-248.

2. K. Brauner, Zur Geometrie der Funktionen Zweier komplexen Veränderlicken, Abh. Math. Sem. Hamburg 6 (1928), 1-54.

3. W. Burau, Kennzeichnung der Schlauchknoten, Abh. Math. Sem. Hamburg 9 (1932), 125-133.

4. G. M. Greuel, Constant Milnor number implies constant multiplicity for quasi-homogeneous singularities, Manuscripta Math. 56 (1986), 159-166.

5. H. Laufer, Tangent cones for deformations of two-dimensional quasi-homogeneous singularities (preprint).

6. Lê Dûng Trâng, Topologie des singularités des hypersurfaces complexes, Astérisque 7, 8 (1973), 171-182.

7. _ Three lectures on local monodromy, Lecture Notes Series No. 43, Matematisk Institut, Aarhus Universitet, Aarhus, 1974.

8. Lê Dûng Trâng and B. Teissier, Report on the problem session, Proc. Sympos. Pure Math., vol. 40, part 2, Amer. Math. Soc., Providence, R. I., 1983, pp. 103-116.

9. M. Lejeune, Sur l'equivalence des singularités des courbes algebroides planes, Coefficients de Newton, Centre de Math. de l'Ecole Polytechnique, 1969.

10. J. Milnor, Singular points of complex hypersurfaces, Ann. Math. Studies, no. 61, Princeton Univ. Press, Princeton, N. J., 1968.

11. J. Milnor and P. Orlik, Isolated singularities defined by weighted homogeneous polynomials, Topology 9 (1970), 385-393.

12. W. Neumann, A calculus for plumbing applied to the topology of complex surface singularities and degenerating complex curves, Trans. Amer. Math. Soc. 268 (1981), 299-344.

13. P. Orlik and P. Wagreich, Isolated singularities of algebraic surfaces with $C^{*}$-action, Ann. of Math. (2) 93 (1971), 205-228.

14. D. O'Shea, Topologically trivial deformations of isolated quasi-homogeneous hypersurface singularities are equimultiple, Proc. Amer. Math. Soc. 101 (1987), 260-262.

15. B. Pérron, Conjugaison topologique des germes de fonctions holomorphe à singularité isolée en dimension trois, Invent. Math. 82 (1985), 27-35.

16. J. Reeve, A summary of results in the topological classification of plane algebroid singularities, Rend. Sem. Mat. Torino 14 (1954/1955), 159-187.

17. A. N. Varchenko, Zeta-function of monodromy and Newton's diagram, Invent. Math. 37 (1976), 253-262.

18. E. Yoshinaga, Topological types of isolated singularities defined by weighted homogeneous polynomials, J. Math. Soc. Japan 35 (1983), 431-436. 
19. O. Zariski, On the topology of algebroid singularities, Amer. J. Math. 54 (1932), 433465.

20. $\_$Some open questions in the theory of singularities, Bull. Amer. Math. Soc. 77 (1971), 481-491.

21. _ General theory of saturation and saturated local rings. II, Amer. J. Math. 93 (1971), 872-964.

Department of MATHEMATiCs, University of Illinois at Chicago, ChICAgo, Illinois 60680 